\title{
Ketones, omega-3 fatty acids and the Yin-Yang balance in the brain: insights from infant development and Alzheimer's disease, and implications for human brain evolution ${ }^{\star}$
}

\author{
Stephen C. Cunnane* \\ Department of Medicine, Université de Sherbrooke, Sherbrooke, QC, Canada
}

Received 21 February 2018 - Accepted 8 March 2018

\begin{abstract}
Optimal brain performance is intimately linked to the brain's Yin and the Yang - the balance between its structure and its energy metabolism. This relationship is clearly exemplified in infant brain development and in Alzheimer's disease, and probably also applies to human brain evolution. In these examples, redundant pathways help achieve this important balance. For instance, the key structural lipid for the brain, docosahexaenoic acid (DHA), is supplied to the infant brain from at last three overlapping sources: (i) milk; (ii) infant's own fat stores and (iii) by some endogenous synthesis from $\alpha$-linolenic acid (ALA) or eicosapentaenoic acid (EPA). On the energy side, glucose is normally the brain's main fuel but under conditions of prolonged starvation, it can be almost totally replaced by the ketone bodies, acetoacetate and $\beta$-hydroxybutyrate. When ketones are present in the blood they spare glucose uptake by the brain because they are actually the brain's preferred fuel and are essential for normal infant brain development. The redundant sources of ketones are long chain fatty acids (including the relatively ketogenic ALA) in infant stores, and medium chain triglycerides (MCT) in milk. Besides infancy, nowhere is the strain on the brain's balance between yin and yang more apparent than in Alzheimer's disease (AD). One of the reasons why attempts to treat $\mathrm{AD}$ have largely failed could well be because chronically inadequate glucose supply to some areas of the brain on the order of $10 \%$ is present in people at risk of $\mathrm{AD}$ long before cognitive decline begins. However, brain ketone uptake is still normal even in moderately advanced AD. Hence, treatments that ignore the brain energy (glucose) deficit in $\mathrm{AD}$ would be predicted to fail, but treatments that attempt to rescue brain fuel availability via ketones would be predicted to have a better chance of succeeding. By analogy to ketones sparing glucose for brain energy metabolism, perhaps ALA or EPA entering the brain can help spare (conserve) DHA for its structural role. If so, it would not necessarily be futile to transport ALA and EPA into the brain just to $\beta$-oxidize the majority afterwards; DHA sparing as well as ketone production could be important beneficiaries.
\end{abstract}

Keywords: brain / brain development / Alzheimer's disease / glucose / ketones / beta-hydroxybutyrate / aging / omega3 fatty acids / alpha-linolenic acid / eicosapentaenoic acid / docosahexaneoic acid

Résumé - Cétones et acides gras omega-3, la balance du Yin et du Yang au niveau cérébral : apport des données acquises sur le développement de l'enfant à la maladie d'Alzheimer et implications dans l'évolution du cerveau humain. Les performances optimales du cerveau sont intimement liées au Yin/Yang cérébral: l'équilibre entre sa structure et son métabolisme énergétique. Ce phénomène s'applique spécifiquement au cours du développement cérébral du jeune enfant et de la maladie d'Alzheimer et très probablement tout au long de l'évolution du cerveau humain. Dans le cas des exemples précités, des voies redondantes permettent d'accéder à cet équilibre. Par exemple, le DHA, clé de la structure lipidique cérébrale, est apporté au cerveau de l'enfant via au moins trois sources concomitantes: (i) le lait; (ii) les réserves lipidiques propres à l'enfant; (iii) enfin, via une synthèse endogène à partir de l'acide $\alpha$-linolénique (ALA) ou de l'acide eicosapentaénoïque (EPA). Sur le plan énergétique, dans des conditions normales, le

\footnotetext{
^ The 2017 Chevreul Medal lecture, Lipids and Brain IV Conference,

Nancy, France, October 10, 2017.

*Correspondence: stephen. cunnane@usherbrooke.ca
} 
glucose reste la source principale, mais dans des conditions de jeûne prolongé, il peut être totalement remplacé par l'utilisation de corps cétoniques : l'acétoacétate et le $\beta$-hydroxybutyrate. Quand les cétones sont présentes dans la circulation sanguine, elles permettent l'économie de consommation de glucose par le cerveau, car elles représentent le carburant préférentiel et sont alors essentielles pour le développement cérébral normal de l'enfant. Les sources parallèles de cétones sont les acides gras à longues chaînes issus du tissu adipeux de l'enfant (y compris l'ALA qui est modérément cétogène) et les triglycérides à chaînes moyennes (MCT) du lait. Au-delà de l'enfance, l'impact du Yin et Yang dans l'équilibre cérébral n'est nullepart ailleurs plus évident que dans la maladie d'Alzheimer (AD). Une des raisons pour lesquelles les essais de traitement de l'AD ont tous échoué pourrait être que l'apport insuffisant de glucose dans certaines régions du cerveau (de l'ordre de $-10 \%$ ) précède le déficit cognitif chez les personnes à risques d'AD. Cependant l'utilisation de cétones par le cerveau est normale, même chez les sujets AD en début de pathologie. Ainsi des traitements qui ne prendraient pas en compte le déficit d'apport énergétique en glucose chez ces sujets sont à risque d'échec, alors que des traitements susceptibles de restaurer l'apport énergétique via les cétones ont potentiellement de meilleures chances d'être efficaces. Par analogie aux cétones permettant l'économie de glucose dans le métabolisme énergétique du cerveau, il est possible que l'ALA et l'EPA (qui sont également captés par le cerveau) permettent de préserver la fonction structurelle du DHA. Ainsi, finalement le transport d'ALA et d'EPA vers le cerveau pour être $\beta$-oxydés ne serait pas nécessairement un métabolisme futile: il pourrait participer à une économie de DHA et de production de cétones qui en deviendraient les principaux bénéficiaires.

Mots clés : cerveau / developpement du cerveau / maladie d'Alzheimer / glucose / cétones / béta-hyroxybutyrate / vieillissement / acides gras oméga-3 / l'acide alpha-linolenique / l'acide eicosapentaenoïque / l'acide docosahexaenoïque

\section{Introduction}

Receiving the Chevreul Medal was a very special honour and a great pleasure for me on a couple of levels. First, I have been fortunate to be able develop strong scientific links with several lipid biologists in France over the past 30 years, so to cap those scientific links with an award for research excellence from SFEL is gratifying. Second, it is very satisfying to know that the scientific concepts we have been working on are worthy of this award. Third, but by no means of the least importance, my mentor and friend of nearly 40 years, Professor Michael Crawford, received the Chevreul Medal at the previous Lipids and Brain meeting in 2015 in Paris. Before him, Dr. Stanley Rapoport and Nicolas Bazan were the recipients at the Lipids and Brain meetings in 2007 and 2011. To me, all three have blazed an extraordinary path in the science of brain lipids, so to be joining them as a recipient of this award is truly wonderful.

What I will try to do here is bring together two parts of a story about brain lipids and brain energy metabolism on which we have been working for many years now. In my view, a complete understanding of brain function, especially in humans, is incomplete without recognizing the essential balance between the brain's Yin (structure) on the one hand, and its Yang (energy metabolism) on the other. Most of the effort expended to understand the role of lipids in brain function in health and disease has gone into the 'structural' theme, i.e. how specialized lipids like docosahexaenoic acid (DHA), cholesterol and many others are involved in optimizing membrane structure, cell signaling and other molecular processes in the brain. But in energetic terms, the human brain is a costly organ to run. There are clear clinical examples that insufficient energy availability to the brain, i.e. problems getting glucose into the brain and/or metabolizing it to produce ATP, compromise brain function and contribute to propagating neurological diseases like Alzheimer's.
We propose that brain development and function in humans cannot be optimal without respecting the Yin-Yang balance, i.e. the need for a specialized membrane structure for neuronal connectivity but the needs at the same time to meet its high energy requirement. Major challenges to acquiring both the structural and the energetic component of mammalian brain function have to be surmounted during infant development, so therefore also had to be surmounted into order to evolve the modern human brain from its primate blueprint.

Redundant pathways contribute to ensuring that the requirements for both these components of optimal brain function are achieved. This review will summarize the case that understanding human brain function from its very origins over two million years ago, to its development in infants today, to its resilience in older age is intimately linked to the overlapping or redundant pathways that help ensure the brain's structural and energy requirements. Successful strategies to treat diseases like Alzheimer's will ultimately involve optimizing this link.

\section{DHA and brain function}

DHA is one of the most studied of the specialized structural lipids in the brain. As gas chromatographic separation and measurement of long chain fatty acids improved in the 1960s, DHA quickly became of interest because of both its relatively high concentration in the brain's grey matter and in the retina, as well as the measurable behavioral and cognitive consequences of experimental dietary omega-3 fatty acid deficiency (at least in experimental animals). It has been clear for many years now that adequate DHA levels in the brain are required for normal mammalian brain development. Still, intense debates about whether DHA should be present in milk formulas arose in the 1980s and 1990s because it was difficult to demonstrate in healthy term infants that a dietary source of DHA was necessary for normal brain development. That difficulty arose because the healthy term infant is well- 
supplied with DHA from overlapping (redundant) sources. Even if it is not consuming breast milk, the healthy term infant still normally has over a gram of DHA in its fat stores that could in theory supply the developing brain with DHA for several months (Cunnane et al., 2000). The infant can also makes some DHA from stored or incoming alpha-linolenic acid (ALA) and eicosapentaenoic acid (EPA). Hence, redundant pathways, i.e. not only DHA intake in breast milk, but also DHA in body stores, as well as some DHA synthesis, are available precisely because DHA is so important for the developing human brain.

Despite this redundancy, optimal brain DHA content is not guaranteed. From my perspective, two key observations drove home the essentiality of pre-formed dietary DHA for normal brain development in human infants:

- brain DHA was much lower in term infants not consuming DHA compared to breast-fed infants (Farquharson 1994; Makrides et al., 1994);

- the brain deficiency of DHA in Zellweger syndrome which is associated with severe functional consequences that could at least partially be alleviated by oral DHA supplementation (Martinez, 1992).

In both of these examples, neither DHA synthesis from ALA nor DHA stores at birth were sufficient to compensate for inadequate DHA intake, so the brain does not accumulate DHA at the level it should and functional deficits related to DHA deficiency occur. DHA synthesis within the body was never intended to ensure sufficient DHA for normal brain development in humans but this limitation was not an issue because until the last century or so, a significant and regular dietary supply of pre-formed DHA from fish and shellfish met the brain's needs.

Nevertheless, many term infants have been raised on formulas containing no DHA and probably became cognitively normal adults. Hence, redundant pathways clearly help protect the brain DHA supply, but they also make research involving omega-3 or DHA deficiency in humans, especially infants, experimentally and ethically more difficult. As a result, we have no really good model of omega-3 deficiency in humans. Not surprisingly therefore, debates still linger - can humans make enough DHA from ALA and EPA; do adults need preformed dietary DHA; DHA is essential in the brain but is it essential in the human diet; are ALA or EPA themselves important for the brain? Studies in omega-3 deficient animals contribute to understanding the brain biology of DHA but these models often exaggerate the consequences of DHA deficiency because experimental dietary omega-3 deficiency in animals can be much more extreme than is plausible in humans, in part because newborn lab animals have no fat stores at birth so have almost no DHA reserves (no redundant sources of DHA) at birth.

It is interesting to compare DHA to another important brain lipid - cholesterol. Despite arguably even greater importance of cholesterol for brain development than for DHA, the strategy to ensure adequate brain cholesterol is totally different than for DHA. Cholesterol is important for brain membrane structure and is also important for Hedgehog expression and normal embryonic development. Transport of cholesterol into the brain is slow and by all accounts inadequate to meet the needs of the developing brain (Jurevics and Morell, 1995). The

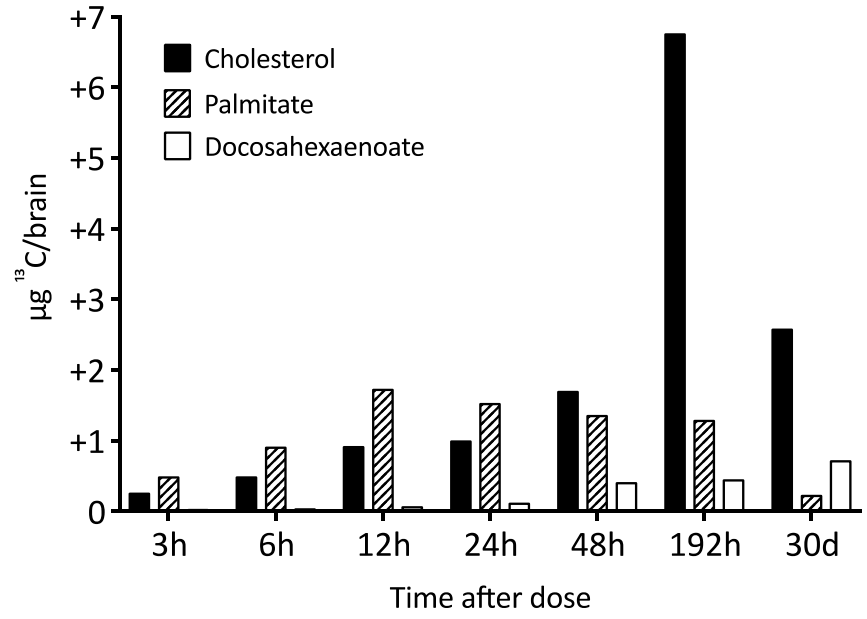

Fig. 1. ${ }^{13} \mathrm{C}$ incorporation into brain total lipids of suckling rats given an oral dose of ${ }^{13} \mathrm{C}-\alpha$-linolenate. ${ }^{13} \mathrm{C}$ incorporation into brain cholesterol and palmitate greatly exceeded incorporation into DHA. These results are taken from a previous publication (Menard et al., 1998), and were corroborated by other reports (Dhopeshwarkar and Subramanian, 1975; Sinclair, 1975; Williard et al., 2001; Chen et al., 2013).

strategy taken by mammals to ensure sufficient brain cholesterol accumulation was to evolve sufficient capacity to synthesize all the cholesterol needed by the brain within the brain itself. At least two different (redundant) substrates are used-glucose and ketones, a point to which we will return. Incidentally, the 'in situ synthesis' strategy for brain cholesterol also applies to brain palmitate, stearate and oleate.

These two different strategies to acquire important brain lipids (pre-formed supply of DHA imported into the brain $v s$. in situ synthesis of cholesterol within the brain) have a point in common because at least one omega- 3 fatty acid contributes to the synthesis of both-ALA.

\section{ALA, ketones and the developing brain}

In one of our experiments in which we were attempting to measure DHA synthesis in the brain of rat pups by in vivo carbon-13 $\left({ }^{13} \mathrm{C}\right)$ nuclear magnetic resonance spectroscopy after an oral tracer dose of ${ }^{13} \mathrm{C}$-ALA, we were thrilled that we could detect the synthesis and accumulation of ${ }^{13} \mathrm{C}$-DHA noninvasively in real time. Indeed, this an accomplishment in its own right. However, we were even more astonished to see that plenty of carbon from ALA ended up in brain lipids other than DHA (Menard et al., 1998) (Fig. 1). Indeed, much more ${ }^{13} \mathrm{C}$ from the tracer $\left({ }^{13} \mathrm{C}\right.$-ALA) was accumulating in the brain as ${ }^{13} \mathrm{C}$-labeled palmitate, stearate, oleate and cholesterol than as ${ }^{13} \mathrm{C}$-DHA! Seeing this occur in a living rat in real time on the screen of an NMR spectrometer was disconcerting and made me realize that it could not be due to artefacts or analytical or other technical errors during sample processing - we were still in vivo - there were no samples yet! Learning that more ALA was metabolized outside compared to within the omega-3 pathway was a game-changer for me because it became clear that viewing ALA's role simply as the 'parent' to the omega-3 pathway was much too narrow a perspective (Fig. 2). It was also a relief to subsequently learn that we were not the first to 


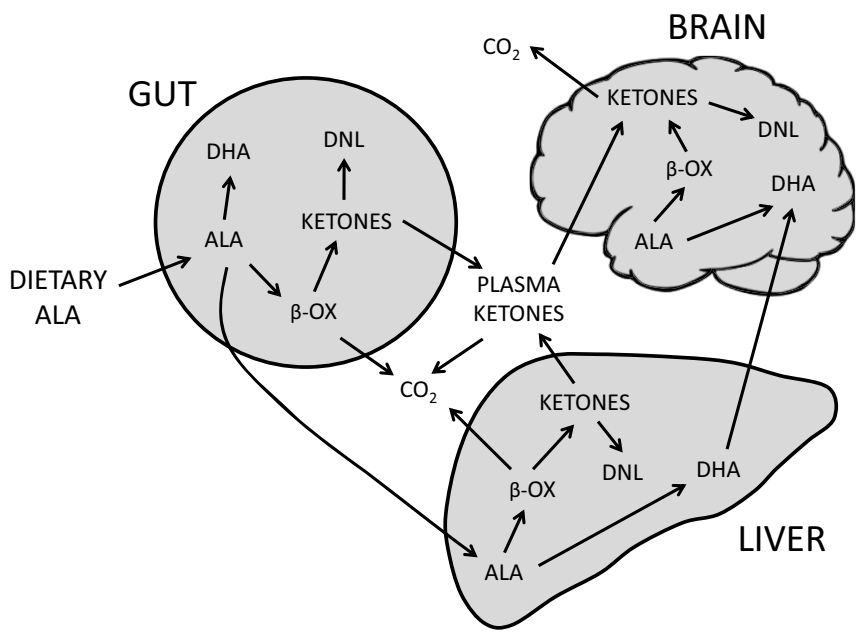

Fig. 2. The multiple metabolic fates of $\alpha$-linoleate (ALA) in the neonate. DHA: Docosahexaenoate; DNL: de novo lipogenesis; $\beta$-OX: $\beta$-oxidation (Dhopeshwarkar and Subramanian, 1975; Sinclair, 1975; Sheaff Greiner et al., 1996, Cunnane et al., 2000; Williard et al., 2001; Cunnane et al., 2003; Chen et al., 2013).

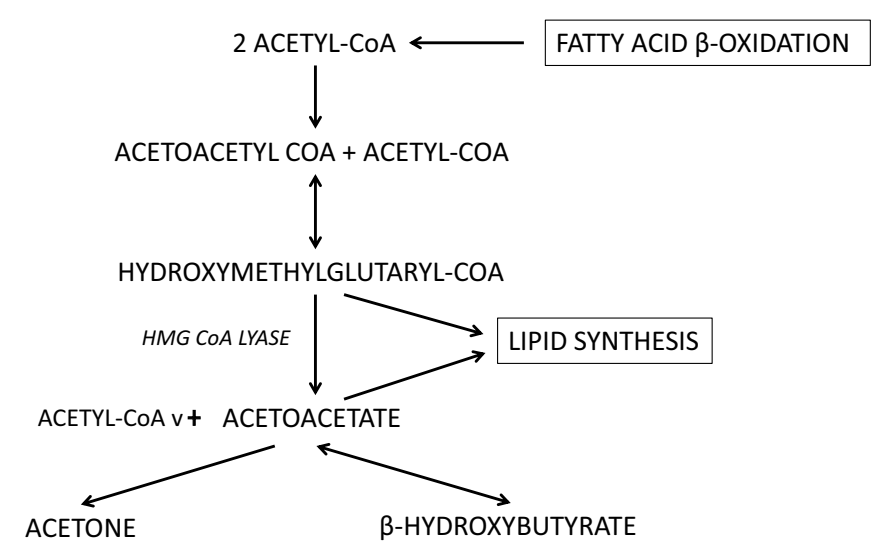

Fig. 3. Ketones are synthesized principally from fatty acids. Acetoacetate and $\beta$-hydroxybutyrate can be use by the brain as an energy substrate whereas acetone is eliminated in the breath.

report carbon recycling of ALA (Dhopeshwarkar and Subramanian, 1975; Sinclair, 1975) and that others were simultaneously finding the same result in different animal models with ALA (Sheaff Greiner et al., 1996). Nevertheless, we were the first to follow up this observation and find that it involved the ${ }^{13} \mathrm{C}$ passing from ALA into de novo lipogenesis via ketone bodies (ketones).

The ketones are beta-hydroxybutyrate, acetoacetate and acetone (Fig. 3). While it is true that plasma ketones in the 15$25 \mathrm{mM}$ range indicate metabolic ketoacidosis, it is totally inaccurate to dismiss the important role of ketones in the more physiological range $(0.5-3 \mathrm{mM})$ on the basis of the risks associated with their high levels in untreated type 1 diabetes. This would be like dismissing the importance of glucose or cholesterol on the basis that high levels lead to type 2 diabetes or increased risk of cardiovascular disease, respectively. Interest in the physiological role of ketones was greatly stimulated by reports that they can meet upwards of $75 \%$ of the brain's energy requirements in adults undergoing medicallysupervised starvation lasting 30-60 days (Owen et al., 1967; Drenick et al., 1972). In humans, ketones are the main replacement brain fuel in the presence of declining glucose availability. The brain appears to be unique in this respect because the main replacement fuel for glucose in other organs is free fatty acids.

Ketones are important because they help the adult brain survive starvation. However, starvation is a state that may or may not arise so, in my view, surviving the risk of starvation was not the most important reason why, during our evolution, ketones became increasingly physiologically linked to brain function. Rather, it is the infant brain that is the real focus of ketone biology because ketones are essential on a daily basis for normal infant brain development (Robinson and Williamson, 1980; Cremer, 1982). It is entirely normal for healthy infants to be constantly in mild ketosis; it is not due to them being sick, underfed or diabetic. Ketones are an obligatory brain energy substrate in human infants because net glucose production in the human infant is insufficient to meet its brain energy requirements (Cremer, 1982; Bougneres et al., 1986; Cunnane and Crawford, 2014). Again, this is the normal situation on a day-to-day basis, not one provoked by starvation, illness or other form of adversity. Brain ketone uptake is also more efficient in the infant than in the adult. Medium chain triglycerides (MCT) in maternal milk are the main substrate for ketogenesis in all mammals. However, the brain is proportionally much smaller in other large mammals so the need for ketones as an additional fuel for the developing brain in other mammals is also lower than in humans (Robinson and Williamson, 1980). At weaning, the supply of MCT from milk abruptly stops in species other than humans. Body fat stores in human infants accumulate some of the MCT consumed from milk thereby prolonging a certain limited supply of ketone substrate for several weeks to months beyond weaning (Sarda et al., 1987).

Ketones supply about $90 \%$ of the carbon to make lipids (cholesterol, saturated and monounsaturated fatty acids) accumulating in the developing brain (Yeh et al., 1977; Jurevics and Morell, 1995; Edmond et al., 1998). Glucose remains a source of carbon for brain lipids but one that is distinctly secondary to ketones (Fig. 4). Again, this points to redundant pathways that ensure that brain lipids synthesized in situ accumulate as needed. Having ketones as an efficient building block for brain lipids spares some glucose for other functions.

A state of nutritional ketosis induced with a very high fat ketogenic diet is the only well-established treatment for refractory epilepsy (Kossoff et al., 2009; Lee and Kossoff, 2011). This has long been known in children but is also true in adults. Hence, a mild-moderate state of ketosis is important for normal brain development and can contribute to normalizing brain function in some diseases such as epilepsy.

Collectively, these observations made ketones of high interest to me but they did not explain why 10-15 times more carbon from ALA was getting into brain cholesterol than into brain DHA. It turns out that ALA is moderately ketogenic (Likhodii et al., 2000; Dell et al., 2001) - not as ketogenic as medium chain fatty acids but more ketogenic than other common dietary long chain fatty acids. ALA is ketogenic because it is the most easily beta-oxidized of the common long 


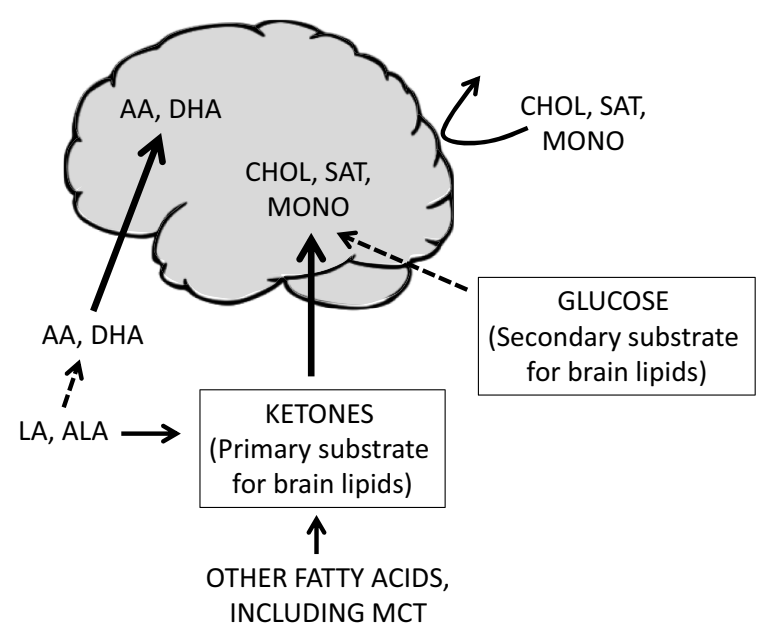

Fig. 4. Origin of brain lipids in neonates. This concept was derived from different sources including the following references: (Patel and Owen, 1976, Yeh et al., 1977, Wells, 1985, Jurevics and Morell, 1995, Edmond et al., 1998, Cunnane et al., 2003, 2011). CHOL: Cholesterol (35-40\% of total); SAT: Palmitate (16:0) and stearate (18:0); MONO:

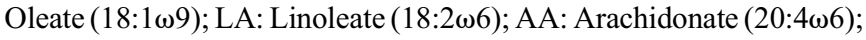

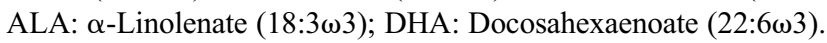

\section{Differential $\beta$-oxidation of long chain fatty acids}

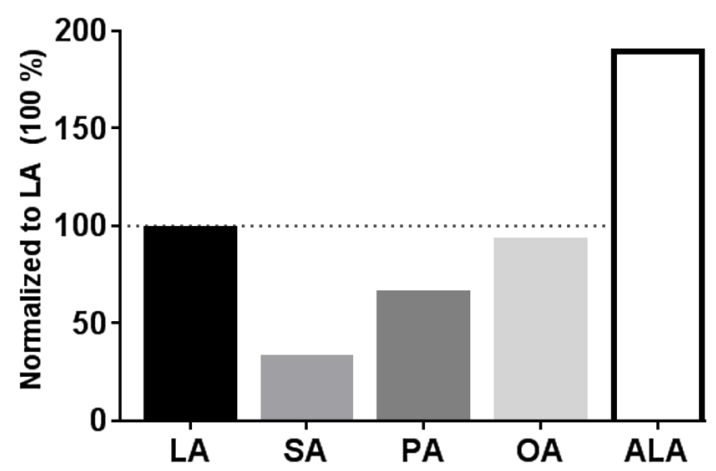

Fig. 5. Differential $\beta$-oxidation of long chain fatty acids (normalized to linoleate $[\mathrm{LA}]-100 \%$ ). Data are pooled from multiple sources and experimental models, including rat liver mitochondria (Bjorntorp, 1968; Clouet et al., 1989; Gavino and Gavino, 1991), in vivo studies in humans (Jones et al., 1985; McCloy et al., 2004), rats (Cenedella and Allen, 1969; Leyton et al., 1987), and catfish (Bandyopadhyay et al., 1982). ALA: $\alpha$-Linolenate; LA: linoleate; SA: stearate; PA: palmitate; OA: oleate.

chain fatty acids for which the order of beta-oxidation in numerous models is - ALA $>$ linoleic acid $\geq$ oleic acid $>$ stearic acid $>$ palmitic acid (Gavino and Gavino, 1991; Cunnane et al., 2003; Fig. 5). Rapid beta-oxidation may be one of the main reasons not only for very low levels of ALA in the brain but also for the low rate of conversion of ALA to DHA.

Still this was puzzling: ALA cannot be made in the body so it is essentially a vitamin - why degrade a vitamin and use the resulting carbon to make other molecules for which many other substrates could be used? We still do not really know the answer but, again, redundant pathways seem to be involved.
Given that ketones are doing something important for the developing brain, it would seem to be advisable to have more than one way to make them. Glucose and cholesterol are important for brain function and there are several ways to make them both. ALA is one of the redundant ways to produce DHA but is also the best fatty acid for ketone synthesis once infants are weaned and no longer consuming MCT from breast milk. Fatty acid beta-oxidation is an unlikely pathway towards meeting the brain's enormous energy requirements in infants and children because brain fatty acid transport is too slow but, in the liver, fatty acid beta-oxidation produces sufficient acetyl CoA that leads to ketogenesis.

Ketones therefore have two important and arguably competing roles in human brain development-they are oxidized as fuels but they are also major brain lipid substrates; ALA contributes to both roles. ALA therefore increases the probability that both exogenous (DHA) and endogenous lipids (cholesterol) accumulate in the brain as they should. As well as contributing to the structural development of brain lipids synthesized in situ, some ALA ends up as ketones that are burned for fuel, so, it also helps the developing brain meet its energy requirements (Fig. 2); little wonder that there is virtually no ALA in the brain! Incidentally, linoleic acid is in essentially the same situation as ALA - low level in the brain, beta-oxidized almost as rapidly as ALA, precursor to an important structural lipid in the brain (arachidonic acid), and carbon recycled into de novo lipid synthesis (Anderson and Connor, 1988; Cunnane et al., 2003).

\section{Ketones and brain energy metabolism}

In relation to its size, the human brain consumes a disproportionate amount of energy. In adults it accounts for $2 \%$ of body weight but consumes $20-23 \%$ of the body's daily energy intake. In newborn infants, the situation is even more extreme - the brain represents about $11 \%$ of body weight but consumes at least $70 \%$ of the infant's energy intake. How in the course of human evolution did it become possible to dedicate $70 \%$ of the incoming dietary energy to only one organ (the brain), the function of which is nowhere nearly adequate to help it survive until several years in childhood? The most plausible explanation for this additional energy source is that humans have fat babies and this fat contributes in a significant way to meeting the energy requirements of the brain from before birth throughout childhood (Fig. 6). Term infants are born with 500-600 grams of subcutaneous body fat, which, with rare exception, is not present on the infants of other terrestrial mammals. Fat starts to accumulate on the human fetus at the beginning of the third trimester and is deposited almost exponentially over following 12-14 weeks (Cunnane and Crawford, 2003, 2014). If gestation does not go to term, it is almost certain that there will be less body fat on the newborn than there should be. Amongst several reasons why pre-term and low birth weight infants are vulnerable to neurodevelopmental delay is their smaller fat reserve, so competition inevitably arises because the energy substrates (glucose and ketones) are the same for thermogenesis as they are for body growth and brain development.

The use of both glucose and ketones as the brain's two main fuels is governed by five principles: First, two distinctly 


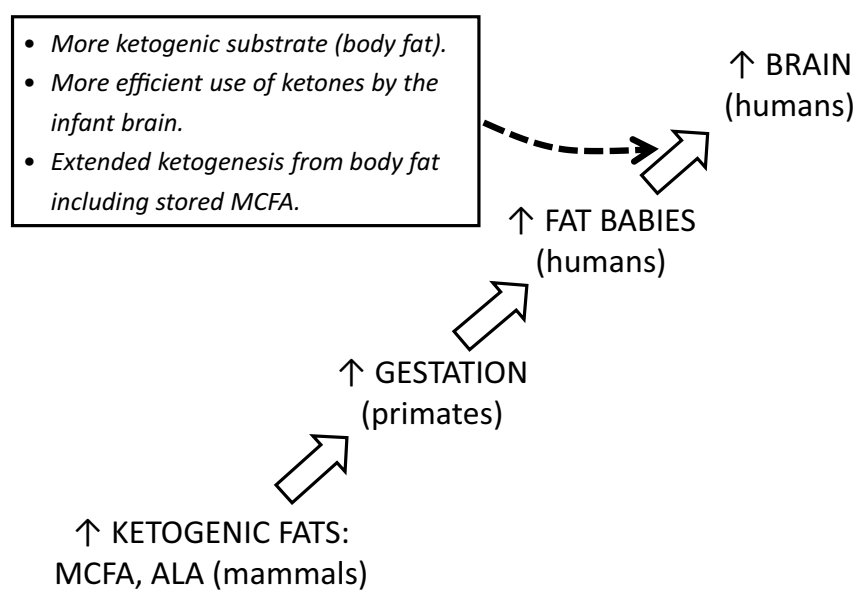

Fig. 6. Setting the metabolic stage for human brain evolution. The capacity to generate ketones from medium chain fatty acids in milk is common to all mammals so would have been a non-specific but essential mammalian feature needed for eventual evolution of the human brain. Prolonged gestation in primates over other mammals would also have favoured brain growth on the fetus but was also not specific to humans. Amongst primates, fat babies are unique to humans and the fat is an energy and nutrient reserve for the brain. Hence, the evolution of fat babies was a crucial to support the energy and structural needs of fetal and neonatal brain expansion in humans. As described in detail elsewhere (Cunnane and Crawford, 2014), a shore-based habitat was a critical source of brain selective nutrients including docosahexaenoate, iodine, iron, zinc, copper, selenium and probably vitamins D and A. ALA: $\alpha$-Linolenate; MCFA: medium chain fatty acids.

different strategies regulate the use of glucose and ketones by the brain, a concept we call 'Push-Pull' (Cunnane et al., 2016a, b). Brain glucose uptake is controlled by brain cell activity. Glucose is transported into the brain via glucose transporters in response to brain cell activation (Pull), so it is largely independent of blood glucose concentration. In contrast, ketones are transported into the brain via monocarboxylic acid transporters directly in response to plasma ketone concentration (Push), not brain activity. Hence, when ketones rise in the blood, they are immediately transported into the brain, an effect not seen with glucose. Second, ketones are transported into the infant brain more rapidly than in adults. In infants, 8 and 10 carbon MCT in breast milk are a major ketone substrate, but MCT are a rare source of ketones in adults unless an MCT supplement is being intentionally consumed. Third, ketones are a minor brain fuel in healthy well-fed adults, i.e. usually $\leq 3 \%$ of brain energy requirements. Nevertheless, ketones are still the adult brain's preferred fuel so they spare brain glucose uptake for other uses (Courchesne-Loyer et al., 2017). Fourth, production of glucose and ketones from endogenous substrates is governed by insulin but insulin appears to have little or no effect on ketone production from exogenous MCT. Fifth, all organs have an alternative fuel to glucose in case of glucopenia, but ketones are not a significant back-up fuel for glucose for any organs other than the brain. Under conditions of severe carbohydrate or food restriction, ketones are produced endogenously from free fatty acids liberated from adipose tissue are used almost exclusively by the brain
(Drenick et al., 1972). This may not be the case with ketones produced from exogenous sources like MCT.

In terms of the brain's opportunistic use of energy substrates, lactate is a good example. Just how much of the brain's energy needs are supplied by lactate and whether that lactate is produced endogenously or exogenously to the brain is controversial (Raichle et al., 1984; Magistretti and Pellerin, 1999; Dienel, 2014; Hyder et al., 2016). It is clear that a surge in lactate supply to the brain caused by intense exercise can be rapidly used as fuel by the brain. A surge in ketone supply (starvation, MCT or other ketogenic condition) can also be rapidly (and preferentially) be used by the brain (CourchesneLoyer et al., 2017). Both situations appear to be opportunistic and both are more useful (essential?) in a large-brained species like humans rather than in other smaller-brained species.

In summary, first impressions about plasma ketones can be deceiving, i.e. that high ketosis is harmful and that ketones are normally only a quantitatively minor brain fuel. Ketones clearly contribute in a major way to brain function, especially in infants. Compared to infants of other mammals, human infants are equipped with a unique supply of ketone substrate in the form of body fat stores. Ketones can be produced from long chain fatty acids in body fat (especially ALA) or from medium chain fatty acids in milk. Thus, ketones are an integral part of intermediary and energy metabolism in humans especially because of our large brain. If ketones are so important for normal human brain function, could safely inducing mild ketosis help restore brain function or delay disease progression?

\section{Keto-neurotherapeutics}

The centenary of the use of the very high fat ketogenic diet for intractable epilepsy will be in 2021(Koppel and Swerdlow, 2017). Hence, long before ketones were known to be the brain's main alternative fuel for glucose, indeed, before any research had begun into the role of ketones in brain function, they were already in clinical use to treat refractory or intractable epilepsy, particularly in children. The very fact that this treatment has not only persisted for a century but has never really been replaced by any new pharmaceuticals is testimony to its therapeutic efficacy. It started with the observation that fasting and a very high fat diet were metabolically similar in that dietary carbohydrate was significantly restricted and that ketones were produced in both conditions. Fasting has been known since biblical times to be beneficial for seizures. However, by definition, fasting is a temporary condition that ends with food being consumed, and only rarely will seizures be totally cured with a few days' fasting. Early in the last century, nutritional science had reached the stage that it was understood that one could use a very high fat (very low carbohydrate) diet to imitate the effect of fasting without actually fasting. The very high fat ketogenic diet treatment of refractory epilepsy continues to be as effective today as it was when it was initiated a century ago. This diet is now also recognized as an effective way to treat severely impaired brain glucose transport due to a mutation in GLUT1. Brain ketone uptake via the monocarboxylic acid transporter is unaffected in GLUT1 deficiency so ketones become an energy lifeline for the brain (Pascual and Ronen, 2015; Mochel, 2017). Because it 
suppresses appetite and decreases the need for insulin, the very high fat ketogenic diet is an effective way to lose weight in obesity and to treat type 2 diabetes. It may also be an excellent adjuvant for the treatment of inoperable brain cancer as well because of the severe dietary carbohydrate restriction, which impairs tumour growth.

The long history of keto-neurotherapeutics led us to ask two questions:

- could ketones be beneficial in Alzheimer's disease?

- could the production of ketones be improved using ALA or eicosapentaenoic acid (EPA)?

Not surprisingly, the focus of most research into the cause of Alzheimer's disease has been on the neuropathology involving plaques and tangles in the brain. Despite intense effort and the attractiveness of this disease being corrected by a drug, the focus on blocking the neuropathology has not yet borne any significant fruit in terms of effective therapeutics. Perhaps this slow progress on the pharmaceutical treatment of Alzheimer's is not surprising because brain glucose uptake has been known to be impaired in Alzheimer's since the 1980s, so brain cells are chronically energy-restricted, which could be contributing to both the neuropathology and cognitive symptoms (Cunnane et al., 2011). Clearly, if brain cells are dying in Alzheimer's disease, they will be consuming less energy and brain glucose (and ketone) uptake will decrease. However, that assumes that the disease causes the brain glucose uptake problem. But what if the brain glucose uptake problem were present before the memory problems - could it is contributing to the memory loss as well as to the plaques and tangles (Fig. 7)? If the problem was specific to glucose, could brain ketone uptake actually still be normal?

In fact, brain glucose uptake is impaired in several conditions associated with increased risk of Alzheimer's disease but decades before its clinical onset. This includes carriers of the Presenilin-1 mutation, carriers of the E4 polymorphism of apolipoprotein E, cognitively normal adults whose parents had Alzheimer's, cognitively healthy older people in general, and some forms of insulin resistance (Cunnane et al., 2016a, b). The simple solution would seem to be to provide more glucose and see whether memory improves. However, brain glucose uptake depends on brain activity not plasma glucose. Furthermore, chronic hyperglycemia actually contributes more to Alzheimer's rather than alleviating it because it contributes to insulin resistance, which is already fairly prevalent in older people and is a major risk factor for Alzheimer's disease (Mullins et al., 2017; Neth and Craft, 2017).

If brain cells are taking up less glucose because they are dying then brain ketone uptake should also be defective in Alzheimer's disease. We developed a method to measure brain ketone uptake by positron emission tomography (PET) using the ketone tracer $-{ }^{11} \mathrm{C}$-acetoacetate. Our PET protocol involves sequentially assessing brain ketone uptake first (because ${ }^{11} \mathrm{C}$ is a short-lived radiotracer) followed by assessing brain glucose uptake with the tracer- $-{ }^{18} \mathrm{~F}$-flurodeoxyglucose. This provides a measure of brain uptake of both fuels within a three hour period in the same person on the same day, which is as comparable an assessment of both brain glucose and ketone metabolism as possible (Roy et al., 2012, 2013; Castellano

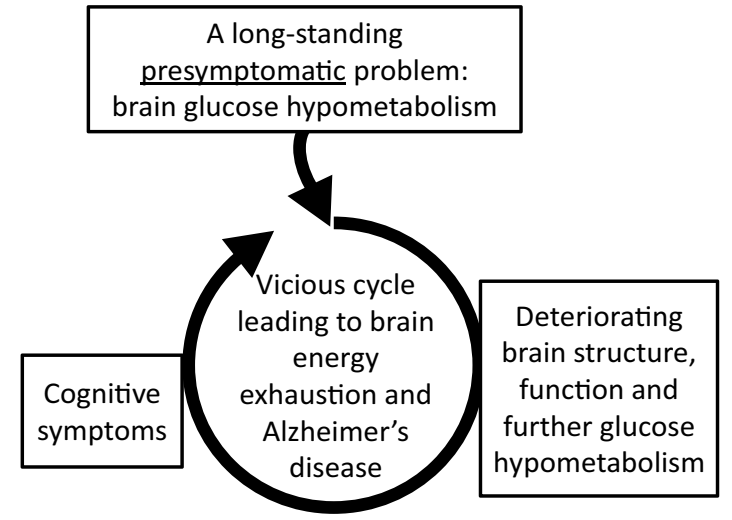

Fig. 7. Brain energy deficit precedes cognitive decline in conditions of increased risk for Alzheimer's disease. Compared to young healthy adults, the regional deficit in brain glucose metabolism is on the order of $8-10 \%$ in those at risk of Alzheimer's but before cognitive symptoms develop, i.e. in healthy older people, young adults with insulin resistance, maternal family history of Alzheimer's, carriers of the presenilin-1 mutation or the E4 allele of apolipoprotein E. Once the brain energy (glucose) deficit is established, the neuropathology develops, leading to cognitive decline, and a vicious cycle involving further brain glucose hypometabolism, more neuropathology, and cognitive deterioration. The process may be interruptible by ketones because brain ketone uptake remains normal in Alzheimer's disease (Cunnane et al., 2016a, b). Clinical trials that are currently in progress will demonstrate whether this concept of keto-neurotherapeutics is valid.

et al., 2017; Courchesne-Loyer et al., 2017; Croteau et al., 2017).

With this dual tracer PET methodology, we have now confirmed many previous studies showing that, compared to healthy older controls, global brain glucose uptake is about $15 \%$ lower in mild-moderate Alzheimer's disease and about 7 $10 \%$ lower in the state known as mild cognitive impairment (MCI) which commonly leads to Alzheimer's (Croteau et al., 2017). Our studies also show that brain ketone uptake is entirely normal in exactly the same Alzheimer or MCI cases that have lower brain glucose uptake (Castellano et al., 2015, 2017; Croteau et al., 2017). So, the good news is that the brain's energy problem in Alzheimer's disease is specific to glucose and does not affect brain ketone uptake. Hence, the argument that brain glucose uptake is impaired in Alzheimer's because the brain cells are dying cannot be correct or they would not be able to metabolize ketones normally. We therefore now need to view Alzheimer's in a different light - it is a metabolic disease in which the brain is starved of its main fuel, glucose (Henderson, 2008; Maalouf et al., 2009; Krikorian et al., 2012; Cunnane et al., 2016a, b; Mamelak, 2017). This energy (glucose) starvation contributes to development of plaques and tangles (Velliquette et al., 2005), neuroinflammation, oxidative stress and undoubtedly to accelerated cell death.

Knowing that brain ketone uptake is still normal in Alzheimer's, the question becomes - could the aging brain be energetically rescued using ketones? In clinical studies starting more than a decade ago, some modest success at improving memory outcomes with a ketogenic approach was reported in both Alzheimer's disease (Henderson, 2008) and in MCI 
(Krikorian et al., 2012). At least three groups including our own have ongoing clinical trials to treat Alzheimer's disease with a 'keto-neurotherapeutic' approach involving either MCT supplements or a ketogenic diet. Results of these trials should be reported in 2018-2019. The value of our PET imaging method is that we are able to quantify the brain glucose deficit early in Alzheimer's disease, which is on the order of 1315 grams/day. This, in turn, permits estimation of the amount of ketones needed to bypass the glucose deficit.

This brings us back to attempts to use omega- 3 fatty acid supplements for the treatment of Alzheimer's. DHA is very slowly beta-oxidized, so, it is not a useful source of ketones but there has long been hope that DHA would be therapeutically beneficial in Alzheimer's because it is so important for cognitive development in children. At the moment, that hope has not been consistently supported by the results of randomized, placebo-controlled trials with fish oils (FreundLevi et al., 2006; Yurko-Mauro and McCarthy, 2010), perhaps in part because brain DHA is not really decreased in Alzheimer's (Cunnane et al., 2012). Indeed, we were optimistic that the importance of DHA for brain glucose transport (Ximenes da Silva et al., 2002) would mean that DHA supplements would help correct the brain glucose uptake problem in Alzheimer's disease. Unfortunately, we did not see an improvement in brain glucose uptake in a pilot trial in older people treated with fish oil (Nugent et al., 2011).

Perhaps the problem is that until the brain's energy deficit is corrected, addressing only the structural or even neuropathological damage in the Alzheimer brain is doomed to fail - the Yin and the Yang need to be corrected together. If so, one could make several predictions:

- a DHA supplement would be more effective in Alzheimer's if accompanied by a source of ketones;

- a ketogenic supplement would be more effective if accompanied by DHA;

- anti-amyloid, anti-tau or anticholinesterase treatments would be more effective if accompanied by a source of ketones (and perhaps DHA);

- a dietary source of ALA, EPA plus DHA would be more beneficial that DHA alone because ALA contributes to ketone production and the brain cholesterol synthesis, and EPA stimulates mitochondrial beta-oxidation via $\operatorname{PPAR} \alpha$, so should help increase the capacity to beta-oxidize fatty acids and produce ketones.

Unfortunately, in our hands neither ALA nor EPA-rich supplements on their own were effective for stimulating ketogenesis in humans (Plourde et al., 2009; Hennebelle et al., 2016). However, they were not tested in Alzheimer's or in combination with a ketogenic supplement like MCT.

\section{Optimizing keto-neurotherapeutics}

Fasting was the first form of keto-neurotherapeutics in ancient times. Fasting is still popular and can be done intermittently or by chronic partial food restriction. It led a century ago to the very high fat ketogenic diet, which induces more sustained ketosis and is clinically effective for intractable epilepsy. MCT have been known to be ketogenic since the 1960s. MCT are approved as a safe food supplement in many countries and have become more widely available to the consumer in the past 10 years. Ketone salts and esters have also recently emerged as ketogenic supplements. Only MCT and very high fat ketogenic diets have so far been tested in Alzheimer's disease or in MCI and both only in short-term studies. The benefits for cognitive function have also been very modest (Henderson et al., 2009; Krikorian et al., 2012), but this may be a dose and/or tolerability issue. It may also depend on the type of MCT being used because MCT are in fact a generic product that can vary significantly in the proportion of 8,10 and 12 carbon fatty acids. It is the 8 carbon fatty acid - caprylic acid - that is the most ketogenic (Vandenberghe et al., 2017b), but most commercially available MCT contain significant amounts of 10, 12 and 14 carbon MCT, which dilute the ketogenic effect of caprylic acid.

Evaluation of memory and cognition requires rigorous study conditions and a relatively large sample size to produce valid results. Other challenges include having an appropriate placebo for MCT, determining an appropriate therapeutic dose and duration for the intervention, achieving an acceptable format for the supplement or ketogenic diet so as to retain the majority of participants, and selecting the appropriate test population, i.e. a primary prevention study in cognitively healthy older people, or secondary prevention in MCI or Alzheimer's. Complimentary interventions that may improve the degree or duration of ketosis include combining MCT with the ketogenic diet (Taylor et al., 2018) or with energy restriction, or adding ketogenic stimulants such as caffeine (Vandenberghe et al., 2017a) and/or exercise (Castellano et al., 2017).

The neuropathology of Alzheimer's disease includes mitochondrial problems, oxidative stress, and neuroinflammation. Oxidative phosphorylation requires functional mitochondria, which is an obligatory part of ketone metabolism to ATP. Since basal brain ketone metabolism is still normal in Alzheimer's, there must be enough functional mitochondria present in the brain to metabolize the ketones. Ketone metabolism is normal across all brain regions that we have studied but it could still be that a large proportion of the mitochondria are still not metabolizing ketones. If mitochondrial function cannot easily be restored to normal, it may be impossible to fully optimize ketogenic treatments for Alzheimer treatment. This field faces a clear opportunity but several significant challenges as well. Further research during the next 2-3 years will demonstrate whether the potential of keto-neurotherapeutics can be realized.

\section{Perspective}

Interestingly, in the absence of a dietary source of DHA, rats in mild nutritional ketosis induced by a very high fat ketogenic diet have higher brain DHA than rats consuming a conventional low fat diet (Taha et al., 2005). The higher brain DHA and increased DHA synthesis on a ketogenic diet was probably due to greater mobilization of fatty acids including ALA and EPA from adipose tissue due to chronically low insulin. DHA is not easily $\beta$-oxidized nor is it ketogenic but, along with ALA and EPA, DHA is one of the fatty acids that is most easily mobilized from adipose tissue (Raclot and Groscolas, 1993). Hence, nutritional ketosis involves multiple 


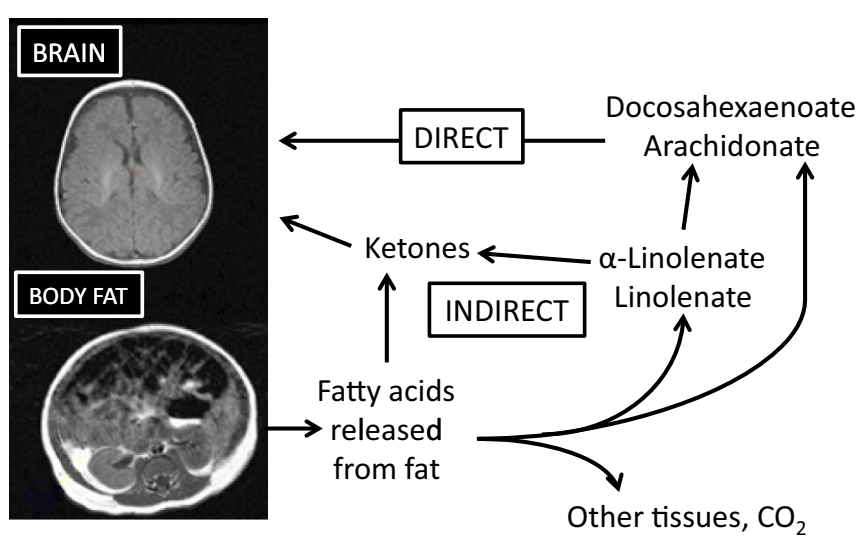

Fig. 8. Conditions of low insulin (fasting, infancy, ketogenic diet) result in mobilization of fatty acids from adipose tissue. Some of the released fatty acids are easily $\beta$-oxidized for energy $(\alpha$-linolenate, linoleate, palmitate, oleate; see Fig. 5) and for ketogenesis (especially ALA). The carbon from these fatty acids reaches the brain indirectly through ketones (INDIRECT route). Other fatty acids released from adipose tissue are much less beta-oxidized and are not ketogenic, i.e. docosahexaenoic acid (Raclot and Groscolas, 1993; Taha et al., 2005) and arachidonic acid. These fatty acids reach the brain directly (DIRECT route). These two processes (fatty acids for ketogenesis for brain energy and fatty acids for brain membrane integrity) contribute to the brain's Yin and Yang; both are needed for optimal brain development in infants and potentially for optimal treatment of certain neurological diseases including epilepsy (Voskuyl, 2002) and Alzheimer's.

metabolic changes that permit adipose tissue to supply not only fatty acids for ketogenesis but also structural fatty acids like DHA to the brain (Fig. 8). This property of nutritional ketosis that contributes to both the brain's Yin and Yang needs further study because it probably applies to understanding healthy infant development and to the treatment of neurological diseases including epilepsy, Alzheimer's, and perhaps others.

The message is threefold: first, an exploratory research project in a pre-clinical model with a new method can lead to an unexpected observation which, if followed up, can lead to a clinical project with a potential treatment for a devastating neurodegenerative disease. In the case of our ${ }^{13} \mathrm{C}$-tracer study to observe ALA conversion to DHA in the brain of rat pups, we demonstrated that ALA is at the intersection of brain energy metabolism and lipid structure (Fig. 2). Both ALA and EPA are poor second choices compared to preformed DHA for supplying brain DHA but may be useful as back-ups. ALA is the best long chain fatty acid at multi-tasking; it has high affinity for carnitine palmitoyl transferase (Gavino and Gavino, 1991), is readily beta-oxidized and is very actively recycled into de novo lipogenesis. The metabolic fate of EPA is less well defined but, like ALA, it too is readily beta-oxidized in the brain (Chen et al., 2013). These observations hopefully contribute to a better understanding of why the levels of ALA and EPA are so low in the brain.

Second, those interested in developing a treatment for Alzheimer's disease should think about nutritional ketosis. Deteriorating brain glucose metabolism is clearly present in older people who are still cognitively normal. Deteriorating brain glucose uptake is also present in young people with insulin resistance, which shows that insulin sensitivity is perhaps the most important modifiable factor to consider in relation to Alzheimer risk. Insulin resistance puts pressure on glucose utilization by the brain long before the cognitive capacity of the aging brain deteriorates (Fig. 7). Ketones and exercise are emerging as the best solutions to this problem. A keto-neurotherapeutic strategy in Alzheimer's is founded on ketones being an essential part of normal brain development in mammals, especially in humans. In simple terms, what applies to successful infant brain development probably applies to successful brain aging. There will be no miracle drugs for Alzheimer's, no one-size-fits-all treatment. It will require a multi-modal solution in which brain energy requirements are being met with ketones in some form or other, plus other interventions including nutrients like DHA, exercise to improve glucose metabolism and cognitive stimulation (Ngandu et al., 2015).

Third, understanding human brain development is the key to understanding human brain evolution. Human brain evolution is an unparalleled success story from a cognitive perspective but there is no free lunch-brain evolution came with risks and vulnerabilities, especially for the infant (Crawford et al., 2014). Redundant nutritional and metabolic pathways reduced those risks but they still remain acutely present today. Providing sufficient brain selective nutrients, especially iodine and iron, remains a huge geo-political challenge in the world today for which, there are no redundant pathways to provide relief. A full understanding of human brain evolution involves an appreciation of the very real nutrient vulnerability in populations that consume little or no fish or shellfish today. The structural refinement of the primate brain as it became the human brain also needed a reliable source of much more energy so as to expand and develop an order of magnitude more synapses. A sustained source of ketones broke through this energetic barrier to cognitive expansion in other primates but only because human infants also evolved fat reserves sufficient to sustain ketogenesis throughout childhood (Cunnane and Crawford, 2014).

Finally, true quantification of biological results is becoming a casualty as computing power increases exponentially and the trend towards 'big data' gathers momentum. For what it is worth, this story would not have been possible without quantification of metabolic data. We simply could not ignore the fact that $10-50$ times more ${ }^{13} \mathrm{C}$ (actual $\mu$ moles) from ALA ended up in de novo lipogenesis compared to DHA (Menard et al., 1998). Much later, we quantified the brain glucose deficit ( $\mu \mathrm{mol} / 100 \mathrm{~g} / \mathrm{min})$ in cognitively healthy older people, MCI and Alzheimer's disease compared to healthy young adults. Without having done so, we could not have estimated the therapeutic target for ketones to bypass the energy deficit (Nugent et al., 2014; Castellano et al., 2015; Cunnane et al., 2016a, b; Croteau et al., 2017). It is laborious and it is more expensive but, in the development of keto-neurotherapeutics, quantification was essential at several levels.

Acknowledgments. I sincerely thank SFEL for the Chevreul medal. I also would like to thank my friends and colleagues in France, most especially Philippe Guesnet, who has collaborated with me on some interesting projects, most notably our studies on 'pure' omega-6 fatty acid deficiency. Thanks also to Michael Crawford for your friendship, 
inspiration and encouragement and, for being present when I received the Chevreul Medal. Thank you to my amazing research team, our enthusiastic participants and our many collaborators who are so much a part of this story. Thank you to Sébastien Tremblay and the Sherbrooke Molecular Imaging Center for rising so splendidly to the challenge of making the ${ }^{11} \mathrm{C}$-acetoacetate; you made so much of this journey possible. Thank you, Monique, for accompanying me all along the way.

Financial and/or material support for our work has been provided by CIHR, NSERC, FRQS, CFI, Canada Research Chairs, Alzheimer Association USA, Mitacs, the Research Center on Aging, Université de Sherbrooke, Sojecci2, Abitec Corp, Accera, Bulletproof, Nestlé, Nisshin OilliO, Prüvit, and Ultragenyx.

\section{References}

Anderson GJ, Connor WE. 1988. Uptake of fatty acids by the developing rat brain. Lipids 23(4): 286-290.

Bandyopadhyay GK, Dutta J, Ghosh S. 1982. Preferential oxidation of linolenic acid compared to linoleic acid in the liver of catfish (Heteropneustes fossilis and Clarias batrachus). Lipids 17(10): 733-740.

Bjorntorp P. 1968. Rates of oxidation of different fatty acids by isolated rat liver mitochondria. J Biol Chem 243(9): 2130-2133.

Bougneres PF, Lemmel C, Ferre P, Bier DM. 1986. Ketone body transport in the human neonate and infant. J Clin Invest 77(1): 42-48.

Castellano CA, Nugent S, Paquet N, et al. 2015. Lower brain 18Ffluorodeoxyglucose uptake but normal 11C-acetoacetate metabolism in mild Alzheimer's disease dementia. J Alzheimers Dis Netherlands 43: 1343-1353.

Castellano CA, Paquet N, Dionne IJ, et al. 2017. A 3-month aerobic training program improves brain energy metabolism in mild Alzheimer's disease: preliminary results from a neuroimaging study. J Alzheimers Dis 56(4): 1459-1468.

Cenedella RJ, Allen A. 1969. Differences between the metabolism of linoleic and palmitic acid: utilization for cholesterol synthesis and oxidation to respiratory CO2. Lipids 4(2): 155-158.

Chen CT, Domenichiello AF, Trepanier MO, Liu Z, Masoodi M, Bazinet RP. 2013. The low levels of eicosapentaenoic acid in rat brain phospholipids are maintained via multiple redundant mechanisms. J Lipid Res 54(9): 2410-2422.

Clouet P, Niot I, Bezard J. 1989. Pathway of alpha-linolenic acid through the mitochondrial outer membrane in the rat liver and influence on the rate of oxidation. Comparison with linoleic and oleic acids. Biochem J 263(3): 867-873.

Courchesne-Loyer A, Croteau E, Castellano CA, St-Pierre V, Hennebelle M, Cunnane SC. 2017. Inverse relationship between brain glucose and ketone metabolism in adults during short-term moderate dietary ketosis: a dual tracer quantitative positron emission tomography study. J Cereb Blood Flow Metab 37(7): 2485-2493.

Crawford MA, Broadhurst CL, Cunnane S, et al. 2014. Nutritional armor in evolution: docosahexaenoic acid as a determinant of neural, evolution and hominid brain development. Mil Med 179 (11): 61-75.

Cremer JE. 1982. Substrate utilization and brain development. $J$ Cereb Blood Flow Metab 2(4): 394-407.

Croteau E, Castellano CA, Fortier M, et al. 2017. A cross-sectional comparison of brain glucose and ketone metabolism in cognitively healthy older adults, mild cognitive impairment and early Alzheimer's disease. Exp Gerontol. DOI: 10.1016/j. exger.2017.1007.1004.

Cunnane SC, Crawford MA. 2003. Survival of the fattest: fat babies were the key to evolution of the large human brain. Comp Biochem Physiol A Mol Integr Physiol 136(1): 17-26.

Cunnane SC, Crawford MA. 2014. Energetic and nutritional constraints on infant brain development: implications for brain expansion during human evolution. J Hum Evol 77: 88-98.

Cunnane SC, Francescutti V, Brenna JT, Crawford MA. 2000. Breastfed infants achieve a higher rate of brain and whole body docosahexaenoate accumulation than formula-fed infants not consuming dietary docosahexaenoate. Lipids 35(1): 105-111.

Cunnane SC, Ryan MA, Nadeau CR, Bazinet RP, Musa-Veloso K, McCloy U. 2003. Why is carbon from some polyunsaturates extensively recycled into lipid synthesis? Lipids 38(4): 477-484.

Cunnane S, Nugent S, Roy M, et al. 2011. Brain fuel metabolism, aging, and Alzheimer's disease. Nutrition (Burbank, Los Angeles County, Calif.) 27(1): 3-20.

Cunnane SC, Schneider JA, Tangney C, et al. 2012. Plasma and brain fatty acid profiles in mild cognitive impairment and Alzheimer's disease. Journal of Alzheimer's disease : JAD 29(3): 691-697.

Cunnane SC, Courchesne-Loyer A, St-Pierre V, et al. 2016a. Can ketones compensate for deteriorating brain glucose uptake during aging? Implications for the risk and treatment of Alzheimer's disease. Ann N Y Acad Sci 3167(1): 12-20.

Cunnane SC, Courchesne-Loyer A, Vandenberghe C, et al. 2016b. Can ketones help rescue brain fuel supply in later life? Implications for cognitive health during aging and the treatment of Alzheimer's disease. Frontiers in Molecular Neuroscience 9 (53): 1-21.

Dell CA, Likhodii SS, Musa K, Ryan MA, Burnham WM, Cunnane SC. 2001. Lipid and fatty acid profiles in rats consuming different high-fat ketogenic diets. Lipids 36(4): 373-378.

Dhopeshwarkar GA, Subramanian C. 1975. Metabolism of linolenic acid in developing brain: I. Incorporation of radioactivity from 1(14)C linolenic acid into brain fatty acids. Lipids 10(4): 238-241.

Dienel GA. 2014. Lactate shuttling and lactate use as fuel after traumatic brain injury: metabolic considerations. J Cereb Blood Flow Metab 34 (11): 1736-1748.

Drenick EJ, Alvarez LC, Tamasi GC, Brickman AS. 1972. Resistance to symptomatic insulin reactions after fasting. $J$ Clin Invest 51 (10): 2757-2762.

Edmond J, Higa TA, Korsak RA, Bergner EA, Lee WN. 1998. Fatty acid transport and utilization for the developing brain. $J$ Neurochem 70(3): 1227-1234.

Farquharson J. 1994. Infant cerebral cortex and dietary fatty acids. Eur J Clin Nutr 48(2): S24-26.

Freund-Levi Y, Eriksdotter-Jonhagen M, Cederholm T, et al. 2006. Omega-3 fatty acid treatment in 174 patients with mild to moderate Alzheimer disease: OmegAD study: a randomized double-blind trial. Arch Neurol 63(10): 1402-1408.

Gavino GR, Gavino VC. 1991. Rat liver outer mitochondrial carnitine palmitoyltransferase activity towards long-chain polyunsaturated fatty acids and their CoA esters. Lipids 26(4): 266-270.

Henderson ST. 2008. Ketone bodies as a therapeutic for Alzheimer's disease. Neurotherapeutics: the journal of the American Society for Experimental NeuroTherapeutics 5(3): 470-480.

Henderson ST, Vogel JL, Barr LJ, Garvin F, Jones JJ, Costantini LC. 2009. Study of the ketogenic agent AC-1202 in mild to moderate Alzheimer's disease: a randomized, double-blind, placebocontrolled, multicenter trial. Nutr Metab (Lond) 6: 31.

Hennebelle M, Courchesne-Loyer A, St-Pierre V, et al. 2016. Preliminary evaluation of a differential effect of an alpha- 
linolenate-rich supplement on ketogenesis and plasma omega-3 fatty acids in young and older adults. Nutrition 32(11-12): 12111216.

Hyder F, Herman P, Bailey CJ, et al. 2016. Uniform distributions of glucose oxidation and oxygen extraction in gray matter of normal human brain: no evidence of regional differences of aerobic glycolysis. J Cereb Blood Flow Metab 36(5): 903-916.

Jones PJ, Pencharz PB, Clandinin MT. 1985. Whole body oxidation of dietary fatty acids: implications for energy utilization. Am J Clin Nutr 42(5): 769-777.

Jurevics H, Morell P. 1995. Cholesterol for synthesis of myelin is made locally, not imported into brain. J Neurochem 64(2): 895-901.

Koppel SJ, Swerdlow RH. 2017. Neuroketotherapeutics: a modern review of a century-old therapy. Neurochem Int s01970186(17): 30227-30229.

Kossoff EH, Zupec-Kania BA, Amark PE, et al. 2009. Optimal clinical management of children receiving the ketogenic diet: recommendations of the International Ketogenic Diet Study Group. Epilepsia 50(2): 304-317.

Krikorian R, Shidler MD, Dangelo K, Couch SC, Benoit SC, Clegg DJ. 2012. Dietary ketosis enhances memory in mild cognitive impairment. Neurobiol Aging 33(2): 425 e 419-427.

Lee PR, Kossoff EH. 2011. Dietary treatments for epilepsy: management guidelines for the general practitioner. Epilepsy Behav 21(2): 115-121.

Leyton J, Drury PJ, Crawford MA. 1987. Differential oxidation of saturated and unsaturated fatty acids in vivo in the rat. Br JNutr 57 (3): 383-393.

Likhodii SS, Musa K, Mendonca A, Dell C, Burnham WM, Cunnane SC. 2000. Dietary fat, ketosis, and seizure resistance in rats on the ketogenic diet. Epilepsia 41(11): 1400-1410.

Maalouf M, Rho JM, Mattson MP. 2009. The neuroprotective properties of calorie restriction, the ketogenic diet, and ketone bodies. Brain Res Rev 59(2): 293-315.

Magistretti PJ, Pellerin L. 1999. Astrocytes couple synaptic activity to glucose utilization in the brain. News Physiol Sci 14: 177182.

Makrides M, Neumann MA, Byard RW, Simmer K, Gibson RA. 1994. Fatty acid composition of brain, retina, and erythrocytes in breast- and formula-fed infants. Am J Clin Nutr 60(2): 189-194.

Mamelak M. 2017. Energy and the Alzheimer brain. Neurosci Biobehav Rev 75: 297-313.

Martinez M. 1992. Abnormal profiles of polyunsaturated fatty acids in the brain, liver, kidney and retina of patients with peroxisomal disorders. Brain Res 583(1-2): 171-182.

McCloy U, Ryan MA, Pencharz PB, Ross RJ, Cunnane SC. 2004. A comparison of the metabolism of eighteen-carbon $13 \mathrm{C}$ unsaturated fatty acids in healthy women. J Lipid Res 45(3): 474-485.

Menard CR, Goodman KJ, Corso TN, Brenna JT, Cunnane SC. 1998. Recycling of carbon into lipids synthesized de novo is a quantitatively important pathway of alpha-[U-13C] linolenate utilization in the developing rat brain. $J$ Neurochem 71(5): 21512158.

Mochel F. 2017. Triheptanoin for the treatment of brain energy deficit: A 14-year experience. $J$ Neurosci Res 95(11): 2236-2243.

Mullins RJ, Diehl TC, Chia CW, Kapogiannis D. 2017. Insulin resistance as a link between Amyloid-Beta and Tau pathologies in Alzheimer's disease. Frontiers in Aging Neuroscience 9(118), in press.

Neth BJ, Craft S. 2017. Insulin resistance and Alzheimer's disease: bioenergetic linkages. Frontiers in Aging Neuroscience 9(345), in press.
Ngandu T, Lehtisalo J, Solomon A, et al. (2015). A 2 year multidomain intervention of diet, exercise, cognitive training, and vascular risk monitoring versus control to prevent cognitive decline in at-risk elderly people (FINGER): a randomised controlled trial. Lancet 385(9984): 2255-2263.

Nugent S, Croteau E, Pifferi F, et al. 2011. Brain and systemic glucose metabolism in the healthy elderly following fish oil supplementation. Prostaglandins Leukot Essent Fatty Acids 85(5): 287-291.

Nugent S, Tremblay S, Chen KW, et al. 2014. Brain glucose and acetoacetate metabolism: a comparison of young and older adults. Neurobiology of aging 35(6): 1386-1395.

Owen OE, Morgan AP, Kemp HG, Sullivan JM, Herrera MG, Cahill GF Jr. 1967. Brain metabolism during fasting. The Journal of clinical investigation 46(10): 1589-1595.

Pascual JM, Ronen GM. 2015. Glucose transporter Type I Deficiency (G1D) at 25 (1990-2015): presumptions, facts, and the lives of persons with this rare disease. Pediatr Neurol 53(5): 379-393.

Patel MS, Owen OE. 1976. Lipogenesis from ketone bodies in rat brain. Evidence for conversion of acetoacetate into acetylcoenzyme A in the cytosol. Biochem J 156(3): 603-607.

Plourde M, Tremblay-Mercier J, Fortier M, Pifferi F, Cunnane SC. 2009. Eicosapentaenoic acid decreases postprandial beta-hydroxybutyrate and free fatty acid responses in healthy young and elderly. Nutrition 25(3): 289-294.

Raclot T, Groscolas R. 1993. Differential mobilization of white adipose tissue fatty acids according to chain length, unsaturation, and positional isomerism. Journal of Lipid Research 34(9): 15151526.

Raichle ME, Herscovitch P, Mintun MA, Martin WR, Powers W. 1984. Dynamic measurements of local blood flow and metabolism in the study of higher cortical function in humans with positron emission tomography. Ann Neurol 15: S48-49.

Robinson AM, Williamson DH. 1980. Physiological roles of ketone bodies as substrates and signals in mammalian tissues. Physiol Rev 60(1): 143-187.

Roy M, Nugent S, Tremblay-Mercier J, et al. 2012. The ketogenic diet increases brain glucose and ketone uptake in aged rats: a dual tracer PET and volumetric MRI study. Brain research 1488: 14 23.

Roy M, Nugent S, Tremblay S, et al. 2013. A dual tracer PET-MRI protocol for the quantitative measure of regional brain energy substrates uptake in the rat. Journal of visualized experiments: JoVE 82: 50761.

Sarda P, Lepage G, Roy CC, Chessex P. 1987. Storage of mediumchain triglycerides in adipose tissue of orally fed infants. $\mathrm{Am} \mathrm{J}$ Clin Nutr 45(2): 399-405.

Sheaff Greiner RC, Zhang Q, Goodman KJ, Giussani DA, Nathanielsz PW, Brenna JT. 1996. Linoleate, alpha-linolenate, and docosahexaenoate recycling into saturated and monounsaturated fatty acids is a major pathway in pregnant or lactating adults and fetal or infant rhesus monkeys. J Lipid Res 37(12): 26752686.

Sinclair AJ. 1975. Incorporation of radioactive polyunsaturated fatty acids into liver and brain of developing rat. Lipids 10(3): 175184.

Taha AY, Ryan MA, Cunnane SC. 2005. Despite transient ketosis, the classic high-fat ketogenic diet induces marked changes in fatty acid metabolism in rats. Metabolism 54(9): 1127-1132.

Taylor MK, Sullivan DK, Mahnken JD, Burns JM, Swerdlow RH. 2018. Feasibility and efficacy data from a ketogenic diet intervention in alzheimer disease. Alzheimer's Dementia: Translational Research Clinical Interventions 4: 28-36. 
Vandenberghe C, St-Pierre V, Courchesne-Loyer A, Hennebelle M, Castellano CA, Cunnane SC. 2017a. Caffeine intake increases plasma ketones: an acute metabolic study in humans. Can J Physiol Pharmacol 95(4): 455-458.

Vandenberghe C, St-Pierre V, Pierotti T, Fortier M, Castellano C-A, Cunnane SC. 2017b. Tricaprylin alone increases plasma Ketone response more than coconut oil or other medium-chain triglycerides: an acute crossover study in healthy adults. Current Developments in Nutrition 1(4): e000257.

Velliquette RA, O'Connor T, Vassar R. 2005. Energy inhibition elevates beta-secretase levels and activity and is potentially amyloidogenic in APP transgenic mice: possible early events in Alzheimer's disease pathogenesis. J Neurosci 25(47): 10874-10883.

Voskuyl RA. 2002. Is marine fat anti-epileptogenic? Nutrition and Health 16(1): 51-53.
Wells MA. 1985. Fatty acid metabolism and ketone formation in the suckling rat. Fed Proc 44(7): 2365-2368.

Williard DE, Harmon SD, Kaduce TL, et al. 2001. Docosahexaenoic acid synthesis from n-3 polyunsaturated fatty acids in differentiated rat brain astrocytes. J Lipid Res 42(9): 1368-1376.

Ximenes da Silva A, Lavialle F, Gendrot G, Guesnet P, Alessandri JM, Lavialle M. 2002. Glucose transport and utilization are altered in the brain of rats deficient in n-3 polyunsaturated fatty acids. J Neurochem 81(6): 1328-1337.

Yeh YY, Streuli VL, Zee P. 1977. Ketone bodies serve as important precursors of brain lipids in the developing rat. Lipids 12(11): 957-964.

Yurko-Mauro K, McCarthy D, Rom D, et al. 2010. Beneficial effects of docosahexaenoic acid on cognition in age-related cognitive decline. Alzheimers Dement 6(6): 456-464.

Cite this article as: Cunnane SC. 2018. Ketones, omega-3 fatty acids and the Yin-Yang balance in the brain: insights from infant development and Alzheimer's disease, and implications for human brain evolution. OCL 25(4): D409. 